\title{
Efecto de la suplementación con Saccharomyces cerevisiae en la producción y calidad de leche en bovinos del Valle de Sibundoy, Putumayo, Colombia
}

\author{
Effect of supplementation with Saccharomyces cerevisiae on milk production and \\ quality in cattle from Valle de Sibundoy, Putumayo, Colombia
Juan Pablo Narváez Herrera ${ }^{1,3}$, Adrián Rolando Riascos Vallejos ${ }^{1}$, José Mauricio Cisneros Montenegro ${ }^{2}$

\section{Resumen}

\begin{abstract}
El objetivo del estudio fue evaluar el efecto de inclusión de dos niveles de Saccharomyces cerevisiae en la producción y calidad de la leche de bovinos del trópico alto del departamento del Putumayo, Colombia. Se utilizaron 12 vacas Holstein a inicios de lactación y de $450 \mathrm{~kg}$ de peso promedio, en un diseño cross over. Las vacas fueron asignadas a dos tratamientos con dos niveles de inclusión de $S$. cerevisiae (T1, $100 \mathrm{~g}$ y T2 $150 \mathrm{~g}$ ) en el suplemento. Se trabajo en dos periodos de 12 días (siete días de adaptación y cinco días de evaluación). Se determinó la calidad y la producción de leche, así como los indicadores del perfil metabólico (glucosa, colesterol y nitrógeno ureico en sangre). No se encontraron diferencias significativas para la producción de leche (14.86 vs $14.27 \mathrm{~kg}$ vaca día) ni en el perfil metabólico, pero hubo una mayor proporción de proteína y grasa en la leche de las vacas de $\mathrm{T} 1(\mathrm{p}<0.05)$.
\end{abstract}

Palabras clave: levaduras, nutrición, rumiantes, suplemento

\footnotetext{
${ }^{1}$ Servicio Nacional de Aprendizaje - SENA, Regional Putumayo; Centro Agroforestal y Acuícola Arapaima, Puerto Asís, Putumayo, Colombia

${ }^{2}$ Centro de Investigaciones en Ciencias Agrarias, Universidad de Nariño, Pasto, Nariño, Colombia

${ }^{3}$ E-mail: jpnarvaez84@misena.edu.co
}

Recibido: 26 de marzo de 2021

Aceptado para publicación: 31 de octubre de 2021

Publicado: 22 de diciembre de 2021

CLos autores. Este artículo es publicado por la Rev Inv Vet Perú de la Facultad de Medicina Veterinaria, Universidad Nacional Mayor de San Marcos. Este es un artículo de acceso abierto, distribuido bajo los términos de la licencia Creative Commons Atribución 4.0 Internacional (CC BY 4.0) [https:// creativecommons.org/licenses/by/4.0/deed.es] que permite el uso, distribución y reproducción en cualquier medio, siempre que la obra original sea debidamente citada de su fuente original 
The aim of this study was to evaluate the effect of inclusion of two levels of Saccharomyces cerevisiae in the production and composition of bovine milk from the high tropics of the department of Putumayo, Colombia. Twelve Holstein cows were used at the beginning of lactation and of $450 \mathrm{~kg}$ of body weight, in a cross-over design. The cows were assigned to two treatments with two inclusion levels of S. cerevisiae (T1, 100 $\mathrm{g}$ and $\mathrm{T} 2,150 \mathrm{~g}$ ) in the supplement. The study was conducted in two periods of 12 days (seven days of adaptation and five days of evaluation). The quality and production of milk were determined, as well as the indicators of the metabolic profile (glucose, cholesterol and blood urea nitrogen). No significant differences were found for milk production (14.86 vs $14.27 \mathrm{~kg}$ cow day) or in the metabolic profile, but there was a higher proportion of protein and fat in the milk of $\mathrm{T} 1$ cows $(\mathrm{p}<0.05)$.

Key words: yeast, nutrition, ruminants, supplementation

\section{INTRODUCCIÓN}

El interés por encontrar aditivos alimentarios inocuos y de bajo costo que permitan maximizar los rendimientos productivos por unidad animal se ha convertido en una necesidad. Una gran variedad de suplementos ha sido utilizada para mejorar la eficiencia de la producción y la función del rumen en el ganado bovino. Entre estos, los antibióticos promotores de crecimiento-APC son sustancias que modifican los procesos digestivos y metabólicos de los animales que se traducen en un incremento en la eficiencia de la utilización de los alimentos (Castro y de Souza Rodríguez, 2008). Sin embargo, los APC han sido restringidos en muchos países por la aparición de cepas resistentes a los antimicrobianos (Briz, 2006). Por otra parte, se han estudiado sustancias con efectos similares sobre el nivel productivo de los animales, como la inclusión de levadura de cerveza (Saccharomyces cerevisiae) que ha evidenciado el aporte de vitaminas, minerales, oligosacáridos, ácidos orgánicos, aminoácidos, péptidos, antioxidantes, y $\beta$ glucanos que mejoran el crecimiento de cierto grupo de bacterias ruminales (Tun et al., 2020).
Rivas et al. (2008) afirman que algunos efectos de la inclusión de $S$. cerevisiae en la dieta de los bovinos se relacionan con la actividad ruminal, tales como la digestibilidad y la degradabilidad de los forrajes, así como con cambios en los patrones de fermentación ruminal (modificación del $\mathrm{pH}$, número y tipo de microorganismos). Galindo et al. (2010) demostraron en estudios in vitro que la inclusión de un hidrolizado enzimático de levadura (S. cerevisiae) tuvo un efecto activador de la población de bacterias celulolíticas del rumen, indicando la posibilidad de que el suministro adecuado de esta levadura incrementa la degradabilidad ruminal en dietas con alto contenido de fibra (Shi et al, 2019).

Aunque son evidentes las ventajas obtenidas tras la utilización de aditivos nutricionales como las levaduras en la alimentación de rumiantes bajo esquemas de alimentación con altos volúmenes de forrajes (García López et al., 2020), algunos datos reportados acerca de la adición de levaduras a dietas de vacas lecheras que evalúan sus efectos sobre la producción y composición de la leche han sido variados e inconsistentes (Rivas et al., 2008). Ante eso, el objetivo del presente 
trabajo fue evaluar el efecto de suministrar un suplemento con diferentes niveles de inclusión de $S$. cerevisiae sobre la producción y composición de la leche de vacas en pastoreo en el trópico alto del departamento del Putumayo, Colombia.

\section{Materiales y Métodos}

\section{Localización}

El estudio se desarrolló entre mayo y diciembre de 2020 en la Finca Villa Clemencia, ubicada en la Vereda San Félix del municipio de Sibundoy, departamento del Putumayo. La zona tiene una altitud de 2100 msnm, $16^{\circ} \mathrm{C}$ de temperatura promedio, $86 \%$ de humedad relativa, $2012 \mathrm{~mm}$ de precipitación anual (IDEAM, 2018). La región corresponde a la zona de vida de bosque húmedo montano bajo bh-MB según Holdridge (1996).

\section{Animales Experimentales}

Se utilizaron 12 vacas Holstein con peso promedio de $450 \mathrm{~kg}$, 90 días en lactancia ( \pm 15) y una producción media de $13 \mathrm{~kg}$ día, distribuidas en dos grupos homogéneos y pastoreando praderas de Lollium perenne de 35 días de edad. Las vacas recibieron los suplementos experimentales elaborados a base de maíz con dos niveles de inclusión de levadura de cerveza (Saccharomyces cerevisiae).

\section{Tratamientos y Diseño Experimental}

Se trabajó con dos niveles de inclusión de $S$. cerevisiae cepa comercial Levapan, utilizada en la industria panificadora, donde los tratamientos experimentales fueron $\mathrm{T} 1$ $(100 \mathrm{~g})$ y T2 $(150 \mathrm{~g})$ en la formulación del suplemento, el cual tenía como base harina de maíz amarillo (híbrido FNC 115) y melaza como edulcorante (Cuadro 1). Se utilizó leche y azúcar como activadores de la levadura. El agua se precalentó a $38{ }^{\circ} \mathrm{C}$ donde se
Cuadro 1. Formulación de los suplementos experimentales

\begin{tabular}{lcc}
\hline Ingredientes & $\mathrm{T} 1$ & $\mathrm{~T} 2$ \\
\hline Maíz harina $(\mathrm{kg})$ & 100 & 100 \\
Levadura $(\mathrm{g})$ & 100 & 150 \\
Melaza $(\mathrm{kg})$ & 6 & 6 \\
Agua $(\mathrm{L})$ & 10 & 10 \\
Azúcar $(\mathrm{g})$ & 300 & 300 \\
Leche fresca $(\mathrm{ml})$ & 300 & 300 \\
\hline
\end{tabular}

diluyó la melaza. Cuando la levadura empezó a crecer se mezclaron todos los materiales hasta obtener una consistencia homogénea. La mezcla se almacenó por $48 \mathrm{~h}$ en un lugar fresco y seco, posteriormente se empacó en sacos de nylon y se llevó a la finca. La cantidad de suplemento suministrada fue de $2 \mathrm{~kg} /$ animal/día y se asumió un consumo de materia seca del $3 \%$ del peso vivo animal (13.5 kg/día de MS).

El efecto de los niveles de inclusión de S. cerevisiae sobre la producción y calidad de leche se evaluó en dos etapas de 12 días, siendo siete días como periodo de adaptación y cinco días para la medición de los resultados, siguiendo la metodología descrita por Castro-Rincón et al. (2020).

En la primera etapa, un grupo de seis vacas recibió el suplemento con $100 \mathrm{~g}$ de $S$. cerevisiae y el otro grupo con $150 \mathrm{~g}$ de $S$. cerevisiae, registrándose en forma individual el consumo de suplemento, y la producción y calidad composicional de la leche. Transcurridos los cinco días de medición se tuvo un periodo de 12 días sin los suplementos, para luego iniciar la segunda etapa, donde los grupos intercambiaron los niveles de suplemento, para repetir la toma de datos. 


\section{Composición Química del Forraje y Su- plementos}

Las muestras del forraje base de la alimentación (Lollium perenne de 35 días) fueron molidas a través de un molino estacionario con una criba de $1 \mathrm{~mm}$ y remitidas al laboratorio de nutrición animal del Centro de Investigación Tibaitata de Agrosavia. Se determinó materia seca (MS), proteína cruda (PC), extracto etéreo (EE), cenizas, fibra detergente neutro (FDN) y fibra detergente ácido (FDA) mediante la técnica de espectroscopía de reflectancia en infrarrojo cercano NIRS (Ariza-Nieto et al., 2018).

La composición química de los suplementos experimentales se determinó según las metodologías establecidas por la AOAC (1995): proteína cruda (PC) por Kjeldahl, cenizas por calcinación a $600{ }^{\circ} \mathrm{C}$, extracto etéreo (EE) por soxhlet, fibra cruda (FC) por el método de Weende.

\section{Producción y Calidad de la Leche}

Se registró la producción de leche al momento de la rutina de ordeño (06:00 y 14:00 h). En cada ocasión, se colectaron muestras de leche de cada vaca para determinar grasa $(\%)$, proteína (\%) y sólidos totales $(\%)$ por medio de un equipo analizador ultrasónico de leche (Milkotester Master Eco).

\section{Perfil Metabólico}

Al inicio y al final de cada periodo de evaluación, previo al primer ordeño del día cuando los animales aún no habían recibido suplemento, se colectaron muestras de sangre $(5 \mathrm{ml})$ de la vena coccígea en tubos Vacutainer. El suero fue obtenido de forma inmediata mediante centrifugación a $3500 \mathrm{rpm}$ durante 8 minutos (Centrifuga Procet Scientific 800-B). El suero fue almacenado en tubos Eppendorf de $1.5 \mathrm{ml}$ y se congeló a $-20{ }^{\circ} \mathrm{C}$ para determinar la concentración de glucosa (técnica de glucosa oxidasa), colesterol (técnica de colesterol oxidasa enzimático de punto final) y nitrógeno ureico en sangre - BUN (Método cinético, kit comercial Spinreact) como indicadores del metabolismo energético y proteico de los animales en estudio.

\section{Análisis Estadístico}

En el estudio se utilizó un diseño de sobre cambio o cross over siguiendo la metodología descrita por Castro-Rincón et al. (2020). Se estimó el efecto del tratamiento y la secuencia de los animales en los dos periodos. Las medias de los tratamientos se ajustaron y compararon según la prueba de Tukey $(p<0.05)$. Para efecto de los análisis estadísticos se utilizó el programa R Project, v. 4.0.3.

\section{Resultados y Discusión}

\section{Composición Química del Forraje y Su- plementos}

La composición química del suplemento experimental y del forraje base de la alimentación (L. perenne de 35 días) se presenta en el Cuadro 2. No se encontraron diferencias significativas en la composición nutricional del suplemento a base de maíz con los dos niveles de inclusión de S. cerevisiae; sin embargo, el incremento en el contenido de PC fue evidente al incluir al maíz amarillo comercial.

Araújo et al. (2008) demostraron que es posible realizar un enriquecimiento proteico de materiales fibrosos (palma forrajera) mediante la inoculación de un cultivo semisólido de Saccharomyces cerevisiae, encontrando que al incluir una concentración del 3\% de inóculo, tras seis horas de incubación el contenido de proteína cruda pasó de 4.4 a $10.4 \%$. En el presente estudio, el contenido de proteína cruda del maíz antes de la inoculación de la levadura fue de $9.6 \%$ mejorando en un $100 \%$ el aporte proteico en el suplemento suministrado a los animales. 
Cuadro 2. Composición química del maíz base del suplemento, de la pastura y de los suplementos conteniendo Saccharomyces cerevisiae como aditivo

\begin{tabular}{lcccc}
\hline Nutrientes & $\begin{array}{c}\text { Maíz (base } \\
\text { del } \\
\text { suplemento) }\end{array}$ & $\begin{array}{c}\mathrm{T} 1 \\
(100 \mathrm{~g})\end{array}$ & $\begin{array}{c}\mathrm{T} 2 \\
(150 \mathrm{~g})\end{array}$ & $\begin{array}{c}\text { Lollium } \\
\text { perenne } \\
\text { 35 días }\end{array}$ \\
\hline Materia seca, MS (\%) & 12.40 & 14.96 & 14.72 & 16.11 \\
Proteína cruda, PC (\%) & 9.60 & 18.75 & 18.69 & 18.13 \\
Fibra cruda, FC (\%) & 1.42 & 1.72 & 2.45 & - \\
Extracto etéreo, EE (\%) & 2.84 & 3.96 & 3.85 & 3.09 \\
Cenizas (\%) & 1.41 & 1.91 & 1.87 & 9.65 \\
Fibra detergente neutro, FDN (\%) & - & - & - & 52.40 \\
Fibra detergente ácido, FDA (\%) & - & - & - & 26.73 \\
\hline
\end{tabular}

Por otra parte, Rompato y Somoza (2015) demostraron que la fermentación en estado sólido de un sustrato compuesto por harina de bagazo de pomelo con Saccharomyces cerevisiae y Bacillus subtilis permite obtener un producto con $16.4 \%$ de PC, contenidos proteicos similares a los encontrados en este estudio. Hernaìndez et al. (2019), al realizar una fermentación de nopal (Opuntia spp) con $1 \%$ urea, $0.1 \%$ de sulfato de amonio y Sacharomyces cereviceae al $1 \%$, encontraron un incremento en el contenido de proteína de 7.08 a $33.5 \%$, demostrando que este tipo de tecnologías de fermentación semisólida se convierten en una excelente alternativa ante la estacionalidad forrajera y el alto costo de fuentes proteicas y suplementos comerciales.

En cuanto a la composición nutricional del suplemento, la inclusión de $100 \mathrm{~g}$ de $S$. cerevisiae resultó ser más eficiente que la inclusión de $150 \mathrm{~g}$, posiblemente debido a la tasa de crecimiento de la levadura en función del tiempo de fermentación realizado. Sería necesario contar con estudios de la cinética de crecimiento de $S$. cerevisiae en mezcla con materias primas alternativas como base de los suplementos, para conocer el nivel de inclusión adecuado.

\section{Producción y Calidad de la Leche}

No se encontraron diferencias estadísticas para la producción de leche vaca/día (14.86 vs 14.27 kg; Cuadro 3). Sin embargo, para las fracciones de proteína y grasa hubo diferencias significativas $(\mathrm{p}<0.05)$ evidenciando ventajas para el tratamiento 1 ( $100 \mathrm{~g} \mathrm{de} S$. cerevisiae). La producción de leche antes del experimento fue de $13.39 \mathrm{~kg} / \mathrm{vaca} /$ día demostrando que la suplementación estratégica con $S$. cerevisiae tuvo un incremento de $1.47 \mathrm{~kg}$ y $0.88 \mathrm{~kg}$ para los tratamientos $1 \mathrm{y}$ 2 respectivamente.

Los resultados de esta investigación coinciden con lo reportado por Rivas et al. (2008), quienes al incluir $10 \mathrm{~g}$ de $S$. cerevisiae en la dieta de bovinos Holstein reportaron un incremento en la producción de leche de 1.57 $\mathrm{kg} / \mathrm{vaca} /$ día. Por otra parte, Suarez-Machín y Guevara-Rodríguez (2017) afirman que el valor medio de mejora en la producción de leche tras incluir levadura en la dieta de los bovinos es del $3.6 \%$ equivalente a $1 \mathrm{~kg} / \mathrm{vaca} /$ día; de igual manera, Salvati et al. (2015) relacionan un incremento de $1.3 \mathrm{~kg} / \mathrm{vaca} /$ día tras incluir $S$. cerevisiae en la dieta de bovinos Holstein durante los meses cálidos del verano en el sudeste de Brasil. 
Cuadro 3. Efecto de la inclusión de dos niveles de Saccharomyces cerevisiae en la producción y calidad de leche (promedio $\pm \mathrm{EE}$ ) de bovinos en pastoreo

\begin{tabular}{lccc}
\hline & $\mathrm{T} 1$ & $\mathrm{~T} 2$ & Valor $\mathrm{p}$ \\
\hline Producción de leche (kg/día) & $(100 \mathrm{~g})$ & $(150 \mathrm{~g})$ & \\
Proteína (\%) & $3.86^{\mathrm{a}} \pm 0.46$ & $14.27^{\mathrm{a}} \pm 0.77$ & 0.7291 \\
Grasa (\%) & $4.18^{\mathrm{a}} \pm 0.04$ & $3.50^{\mathrm{b}} \pm 0.04$ & 0.0117 \\
Sólidos totales $(\%)$ & $13.38^{\mathrm{a}} \pm 0.12$ & $4.00^{\mathrm{b}} \pm 0.04$ & 0.0319 \\
\hline
\end{tabular}

$a, b$ Medias dentro de la misma fila con distinta letra indican diferencias significativas $(p<0.05)$

García López et al. (2020), utilizando la cepa L25/7/13 de $S$. cerevisiae perteneciente a la colección del Instituto Cubano de Derivados de la Caña de Azúcar (ICIDCA), demostraron que la inclusión de $10 \mathrm{~g} / \mathrm{l}$ de levaduras mejoró la calidad composicional de la leche, incidiendo principalmente sobre la proteína láctea reportando valores de $3.2 \%$, aunque inferiores a los encontrados en este estudio ( 3.73 y $3.50 \%$ para $\mathrm{T} 1$ y T 2 , respectivamente). Por otro lado, Desnoyers et al. (2009) aseguran que el aumento de la producción de leche suele estar relacionado con una disminución en el contenido de la grasa; sin embargo, los resultados aquí presentados demuestran que la suplementación con $S$. cerevisiae aumentó la producción de leche sin afectar la composición grasa ni loa sólidos totales.

Valenciaga Gutiérrez et al. (2019) evaluaron el efecto del hidrolizado enzimático de levadura $S$. cerevisiae en la cinética de degradación ruminal, encontrando un incremento en la degradabilidad de la materia seca y, por lo tanto, un mejor aprovechamiento del forraje. Asimismo, Galindo et al. (2010) demostraron que $S$. cerevisiae tiende a reducir la población de metanógenos, abriendo una posibilidad de utilizar este tipo de compuestos para mejorar la eficiencia de la utilización de la energía en los rumiantes, lo cual podría contribuir a mitigar la emisión de gases de efecto invernadero como el metano, contribuyendo de manera positiva con el medio ambiente.

\section{Perfiles Metabólicos}

No se encontraron diferencias estadísticas entre tratamientos para las tres variables en evaluación: glucosa, BUN y colesterol (Cuadro 4). Los resultados de glucosa encontrados se hallan dentro de los estándares nacionales para animales con aptitud lechera (Cubillos, 1999); sin embargo, superiores a los reportados por Campos et al. (2007) y Carizi Cherobin et al. (2019) para vacas Holstein en condiciones tropicales en Colombia y Bolivia, respectivamente. El incremento en la concentración de glucosa podría deberse al elevado aporte energético adicional de los suplementos experimentales.

Los niveles de colesterol se sitúan dentro de los valores de referencia intermedios según Ceballos et al. (2002), los cuales oscilan entre 2.6 y $4.5 \mathrm{mmol} / 1$ para vacas lecheras en fase de lactancia. No obstante, la suplementación con $S$. cerevisiae reportó niveles superiores a los referidos por Sánchez et al. (2014) de $3.23 \mathrm{mmol} / \mathrm{l}$ para vacas lecheras bajo un modelo de semiestabulación. 
Cuadro 4. Indicadores metabólicos de los bovinos suplementados con dos niveles de Saccharomyces cerevisiae

\begin{tabular}{lccc}
\hline & T1 & T2 & Valor $\mathrm{p}$ \\
\hline Glucosa $(\mathrm{mmol} / \mathrm{L})$ & $4.30^{\mathrm{a}} \pm 0.31$ & $(150 \mathrm{~g})$ & 0.9198 \\
BUN $(\mathrm{mmol} / \mathrm{L})$ & $3.07^{\mathrm{a}} \pm 0.19$ & $2.92^{\mathrm{a}} \pm 0.31$ & 0.19 \\
Colesterol $(\mathrm{mmol} / \mathrm{L})$ & $4.22^{\mathrm{a}} \pm 0.40$ & $3.80^{\mathrm{a}} \pm 0.40$ & 0.5037 \\
\hline
\end{tabular}

Medias con una letra común no son significativamente diferentes $(p>0.05)$

La concentración de nitrógeno ureico en sangre (BUN) para los dos tratamientos experimentales se encontró dentro de los valores de referencia para vacas en lactación, los cuales no deben ser superiores a 6.94 mmol/1 (Castro-Rincón et al., 2020). Concentraciones de BUN dentro de los rangos normales indican un balance en el suministro de proteína cruda en la dieta, lo cual no afecta el equilibrio de nitrógeno y péptidos a nivel ruminal.

\section{Conclusiones}

- La suplementación con Saccharomyces cerevisiae tuvo un efecto positivo sobre la producción y calidad composicional de la leche al incluirse en concentración de $100 \mathrm{~g}$ en la formulación del suplemento experimental.

- El perfil metabólico no fue afectado ni presentó diferencias entre los dos niveles de inclusión.

\section{Agradecimientos}

Los autores expresan su agradecimiento al Servicio Nacional de Aprendizaje SENA, Regional Putumayo y al Sistema de Investigación, Desarrollo Tecnológico e Innovación SENNOVA, por los recursos para la ejecución del proyecto de investigación titulado
«Efecto de la suplementación con levadura (Saccharomyces cerevisiae) sobre el comportamiento productivo y emisión de gases de efecto invernadero en bovinos lecheros»».

\section{Literatura Citada}

1. AOAC. 1995. Official methods of analysis of AOAC International. $16^{\text {th }} \mathrm{ed}$, Arlington Virginia USA.

2. Araújo LF, Silva FLH, Brito EA, Oliveira S, Santos ES. 2008. Enriquecimento protéico da palma forrageira com Saccharomyces cerevisiae para alimentação de ruminantes. Arq Bras Med Vet Zoo 60: 401-407. doi: 10.1590/S010209352008000200019

3. Briz RC. 2006. Retirada de los antibióticos promotores de crecimiento en la unión europea: causas y consecuencias. España: Asociación Española de Ciencia Avícola. [Internet]. Disponible en: https://www.wpsa-aeca.es/articulo.php?id_articulo $=362$

4. Campos $R$, Cubillos $C$, Rodas ÁG. 2007. Indicadores metabólicos en razas lecheras especializadas en condiciones tropicales en Colombia. Acta Agron 56: 85-92.

5. Cherobin VC, Garzón JP, Alvarado JP, Marini PR. 2019. Condición corporal y su relación con producción láctea, reproducción y perfil metabólico en vacas le- 
cheras del trópico boliviano. Rev Inv Vet Perú 30: 107-118. doi: 10.15381/rivep.v30i1.14459

6. Castro M, de Souza F. 2005. Levaduras: probióticos y prebióticos que mejoran la producción animal. Cienc Tecnol Agropec 6: 26-38.

7. Castro-Rincón E, Cardona-Iglesias JL, Hernández-Oviedo F, ValenzuelaChiran M. 2020. Efecto del ensilaje de Avena sativa L en la productividad de vacas lactantes en pastoreo. Pastos y Forrajes 43: 150-158.

8. Ceballos A, Gómez PM, Vélez ML, ViIla NA, López LF. 2002. Variación de los indicadores bioquímicos del balance de energía según el estado productivo en bovinos lecheros de Manizales, Colombia. Rev Colomb Cienc Pec 15: 13-25.

9. Cubillos C. 1999. Perfil metabólico en diferentes grupos raciales bovinos lecheros ubicados en el valle geográfico del río Cauca. Tesis de Grado. Palmira, Colombia: Univ. Nacional de Colombia. 110 p.

10. Desnoyers $M$, Giger-Reverdin $S$, Bertin G, Duvaux-Ponter C, Sauvant $D$. 2009. Meta-analysis of the influence of Saccharomyces cerevisiae supplementation on ruminal parameters and milk production of ruminants. J Dairy Sci 92: 1620-1632. doi: $10.3168 /$ jds.20081414

11. Galindo J, Díaz A, González N, Sosa A, Marrero Y, Aldana AI, Noda A. 2010. Effect of hydrolized enzymatic product of Saccharomyces cerevisiae yeasts on the ruminal microbial population with substrate of Pennisetum prupureum vc. Cuba CT-115 under in vitro conditions. Cuban J Agr Sci 44: 275-279.

12. García R, Marrero Y, Galindo J, Moreira O, González M, Noda A. 2020. Efecto de la inclusión de $S$. cerevisiae en la producción de leche y población microbiana ruminal. Livestock Res Rural Develop 32(12). [Internet]. Disponible en: http://www.lrrd.org/lrrd32/ 12/ymarr32192.html
13. Hernaìndez AF, Rodríguez FJM, Herrera CM, Herrera GG, Arriaga OE, Salazar JO, Bautista CH. 2019. Fermentacioìn semisólida del nopal (Opuntia spp) para su uso como complemento proteico animal. Rev Geografía Agríc 63: 87-100. doi: 10.5154/r.rga.2019.63.04

14. Holdridge LR. 1996. Ecología basada en las zonas de vida. $5^{\circ}$ ed. San José de Costa Rica: Instituto Interamericano de Cooperación para la Agricultura. $216 \mathrm{p}$.

15. [IDEAM] Instituto de Hidrología, Meteorología y Estudios Ambientales. 2018. Subdirección de meteorología. [Internet]. Disponible en: http://bart.ideam.gov.co/indiecosistemas/ind/ precipitacion.html

16. Rivas J, Díaz T, Hahn M, Bastidas P. 2008. Efecto de la suplementación con Saccharomyces cerevisiae sobre la producción de leche al inicio de la lactancia en vacas lecheras. Zootec Trop 26: 421428.

17. Rompato KM, Somoza SN. 2015. Enriquecimiento proteico de subproductos frutícolas mediante fermentación en estado sólido con Saccharomyces cerevisiae y Bacillus subtilis. Biotecnol Apl 32: 4221-4227.

18. Salvati GG, Morais Júnior NN, Melo AC, Vilela RR, Cardoso FF, Aronovich M, Pereira RA, et al. 2015. Response of lactating cows to live yeast supplementation during summer. J Dairy Sci 98 : 4062-4073. doi: 10.3168/jds.2014-9215

19. Sánchez JL, Tagle N, Daetz R, Duvauchelle E, Noro M. 2014. Producción láctea e indicadores energéticos en vacas lecheras semiestabuladas tratadas con bajas dosis de somatotropina recombinante bovina durante el período de transición. Rev Cient-Fac Cien V 24: 172-179.

20. Shi W, Knoblock CE, Yoon I, Oba M. 2019. Effects of supplementing a Saccharomyces cerevisiae fermentation product during the transition period on 
rumen fermentation of dairy cows fed fresh diets differing in starch content. J Dairy Sci 102: 9943-9955. doi: 10.3168/ jds.2019-16671

21. Suárez-Machín C, GuevaraRodríguez CA. 2017. Levadura Saccharomyces cerevisiae en la alimentación de rumiantes. Revisión bibliográ- fica. ICIDCA Sobre los Derivados de la Caña de Azúcar 51(2): 21-30. https:// www.redalyc.org/pdf/ $2231 /$ 223154251004.pdf

22. Tun HM, Li S, Yoon I, Meale SJ, Azevedo PA, Khafipour E, Plaizier JC. 2020. Saccharomyces cerevisiae fermentation products (SCFP) stabilize 